\title{
Perfil epidemiológico das intoxicações exógenas no Piauí nos anos de 2013 a 2017
}

\author{
Epidemiological profile of exogenous intoxications in Piauí from 2013 to 2017
}

Perfil epidemiológico de las intoxicaciones exógenas en Piauí en los años de 2013 a 2017

Ellen Soares Ferreira da Silva', Wélida Régia Messias Sousa ${ }^{1}$, Juliana Sousa Soares ${ }^{1}$, Kécya Patricia Costa Macêdo ${ }^{1}$, Bianca de Sousa Leal ${ }^{1}$, Denilson Araújo Oliveira ${ }^{2}$, Sufia de Jesus Costa ${ }^{3}$, Roseane Mara Cardoso Lima Verde 4 , Leonardo Ferreira Soares ${ }^{5}$, Francisco das Chagas Araújo Sousa ${ }^{6}$, Evaldo Hipólito de Oliveira ${ }^{7 *}$, Raimundo Nonato Cardoso Miranda Júnior ${ }^{1}$.

\begin{abstract}
RESUMO
Objetivo: Avaliar as notificações compulsórias de intoxicações exógenas no Piauí nos anos de 2013 a 2017. Métodos: Foi realizada uma pesquisa de cunho exploratório quantitativo, usando as notificações compulsórias de intoxicação exógena do Piauí informada pelo Centro de Informação Toxicológica (CITOX) ao SINAN (Sistema de Informação de Agravos de Notificação) e DATASUS (Departamento de Informática do SUS) nos anos de 2013 a 2017. Resultados: O estudo evidenciou que as intoxicações são um grave problema de saúde pública, tendo maior incidência no ano de 2016, com 1.139 casos, em perímetro urbano, entre jovens do sexo feminino, com faixa etária predominante de 20 a 39 anos, sendo os medicamentos os principais agentes tóxicos utilizados e as tentativas de suicídio as circunstâncias mais prevalentes. Conclusão: Nos anos de 2013 a 2017 foram registrados cerca de 6000 casos de intoxicação exógena no Piauí. Esses dados apontam a importância de medidas de prevenção e estímulo à saúde, planos de combate ao suicídio e demonstram a necessidade de um maior enfoque na prevenção de intoxicações.
\end{abstract}

Palavras-chave: Intoxicação Exógena, Medicamentos, Suicídio.

\begin{abstract}
Objective: To evaluate the compulsory notifications of exogenous intoxication in Piauí from 2013 to 2017. Methods: This was a quantitative exploratory research, using compulsory notifications of exogenous intoxication of Piauí reported by Centro de Informação Toxicológica (CITOX) to SINAN (Sistema de Informação de Agravos de Notificação) and DATASUS (Departamento de Informática do SUS) from 2013 to 2017. Results: The study evidenced that intoxications are a serious public health problem, with a higher incidence in 2016, with 1.139 cases in urban perimeter, among young women aged 20 to 39 years, and the medicines were the main toxic agents used and suicide attempts was the most prevalent circumstances. Conclusion: From 2013 and 2017, about 6000 cases of exogenous intoxication were reported in Piauí. These data show the importance of prevention and health promotion interventions, plans to combat suicide and demonstrate the need for a greater focus on the prevention of intoxication.
\end{abstract}

Palavras-chave: Exogenous Intoxication, Medicines, Suicide.

${ }^{1}$ Centro Universitário Santo Agostinho (UNIFSA), Teresina - Piaui.

${ }^{2}$ Faculdade Maurício de Nassau (UNINASSAU), Parnaíba - PI.

3Instituto Brasil de Pós-Graduação (IBRAS), Teresina - PI.

${ }^{4}$ Programa de Pós-Graduação em Engenharia Biomédica - Universidade Brasil, São Paulo - SP.

${ }^{5}$ Universidade Estadual da Paraíba (UEPB), João Pessoa - PB.

6 Universidade Estadual do Piauí (ESPI), Teresina - PI.

7Universidade Federal do Piauí (UFPI), Teresina - PI. *E-mail: evaldohipolito@gmail.com 


\section{RESUMEN}

Objetivo: Evaluar las notificaciones obligatorias de intoxicaciones exógenas en Piauí en los años de 2013 a 2017. Métodos: Se realizó una investigación de cuño exploratorio cuantitativo, usando las notificaciones obligatorias de intoxicación exógena de Piauí informada por el Centro de Información Toxicológica (CITOX) al SINAN (a) El estudio evidenció que las intoxicaciones son un grave problema de salud pública, teniendo mayor incidencia en el año 2016, con 1.139, en el año 2016, en los años de 2013 a 2017. Resultados: El estudio evidenció que las intoxicaciones son un grave problema de salud pública, teniendo mayor incidencia en el año 2016, con 1.139 en el perímetro urbano, entre jóvenes del sexo femenino, con un grupo de edad predominante de 20 a 39 años, siendo los medicamentos los principales agentes tóxicos utilizados y los intentos de suicidio las circunstancias más prevalentes. Conclusión: En los años de 2013 a 2017 se registraron cerca de 6000 casos de intoxicación exógena en Piauí. Estos datos apuntan a la importancia de medidas de prevención y estímulo a la salud, planes de combate al suicidio y demuestran la necesidad de un mayor enfoque en la prevención de intoxicaciones.

Palavras-chave: Intoxicaciones Exógenas, Medicamentos, Suicidio.

\section{INTRODUÇÃO}

A toxicologia está intimamente relacionada com a história da humanidade e exerce grande influência sob o processo saúde-doença, estando direcionada ao cuidado à saúde do homem e à qualidade do meio em que vive. Segundo Moreira CDAS, et al. (2010), no cenário contemporâneo, o avanço industrial e tecnológico desencadeou o aparecimento de milhares de substâncias tóxicas capazes de provocar danos à saúde dos seres vivos, somadas àquelas que já existiam naturalmente (medicamentos, cosméticos, produtos químicos, de higiene e limpeza, plantas tóxicas, peçonhas, etc.).

A intoxicação humana abrange vários processos fisiopatológicos relacionados com a interação entre um agente de natureza química ou biológica e o organismo. Magalhães AFA (2017) aponta que a expressão da toxicidade dependerá de inúmeros fatores que incluem as propriedades físico-químicas do agente tóxico, as condições de exposição e, fundamentalmente, de uma série de eventos que ocorrem dentro do organismo, ou seja, de seu comportamento toxicocinético e toxicodinâmico.

Dentre as causas de intoxicação exógena estão acidentes com produtos de limpeza, agrotóxicos, animais peçonhentos, plantas tóxicas, pesticidas e inseticidas, drogas de abuso e, por último, uso de medicamentos, que constituem a causa principal. É importante destacar que nem todos esses eventos ocorrem por acidente, dando-se, muitas vezes, por tentativas de autoextermínio, segundo o Centro de Informação Toxicológica do Piauí (CITOX, 2018).

$\mathrm{Na}$ maioria das vezes as intoxicações exógenas se associam ao consumo acidental, proposital ou por dosagem elevadas de substâncias químicas como agrotóxicos, raticidas, pesticidas, drogas de abuso e medicamentos. Chaves LHS, et al. (2017) afirma que as manifestações clínicas de uma intoxicação são variadas, podendo ser a longo, médio e curto prazo, sendo seus efeitos leves como uma reação alérgica, médios quando atingem um órgão e graves quando causam dependência ou levam ao óbito.

Segundo Mendonça REB (2012), as crianças e idosos são os grupos mais vulneráveis a intoxicações essa vulnerabilidade se dá por alguns fatores como: pouco conhecimento quanto ao uso, fácil acesso, uso inadequado, armazenamento incorreto, uso indevido, automedicação e a falta de orientação sobre o produto adquirido.

A falta de conhecimento acerca de eventos toxicológicos e, consequentemente, da identificação dos sinais e sintomas de uma intoxicação exógena bem como as ações tomadas diante disso levam à inadequada investigação e a não correlação desse quadro a uma intoxicação. Diante dessa realidade, esses eventos subnotificados dificultam a obtenção de um índice real para levantamento estatístico das notificações. O Ministério da Saúde (2018) lança em 9 de junho de 2014 a Portaria GM/MS № 1.271, que define a Lista 
Nacional de Notificação Compulsória de doenças, agravos e eventos de saúde pública nos serviços de saúde públicos e privados em todo o território nacional. Essa portaria revoga a de no 104 , de 25 de janeiro de 2011 e define que as intoxicações exógenas são eventos de notificação compulsória semanal.

Segundo Getter CJ, et al (2011), desde 1980, com a criação do Sistema Nacional de Informação TóxicoFarmacológicas (SINITOX) pelo Ministério da Saúde, as emergências tóxicas passaram a ser acompanhadas e desde 1985, passaram a ser divulgadas anualmente as estatísticas sobre esses dados pelos Centros de Assistência em todo o país, centros esses que visam controlar as intoxicações e envenenamentos e que vêm ganhando crescente reconhecimento como importantes fontes de informações emergenciais tóxicas.

Dados do SINITOX (2018) estimam que dos quase 40000 casos de intoxicações em 2016 no Brasil, cerca de $40 \%$ está relacionado ao uso de medicamentos. Porém, esse número pode ser ainda maior levando em consideração que diversos casos não são registrados pelos Centros de Informação e Assistência Toxicológica Estaduais, ou quando registrados não são enviados ao Sistema Nacional de Informação TóxicoFarmacológicas.

Magalhães AFA (2017) destaca que as intoxicações exógenas ainda representam um problema de saúde pública em decorrência do número elevado de casos, podendo ocorrer a nível ocupacional ou domiciliar e geralmente são ocasionados acidentalmente ou por uso indiscriminado de substâncias como medicamentos, domissanitários e pesticidas.

Em razão do índice elevado de intoxicações exógenas relacionadas principalmente a medicamentos, destaca-se a importância das notificações compulsórias desse tipo de evento, que contribui significativamente tanto para a obtenção de dados estatísticos, de um perfil sociodemográfico, bem como para a prevenção, educação e identificação dos sinais e sintomas. Vale ressaltar que o farmacêutico é um profissional importante nesse processo, atuando na prevenção, identificação, tratamento dessas situações de risco e promoção de saúde.

O objetivo desta pesquisa foi avaliar as notificações compulsórias por intoxicação exógenas e traçar um perfil sociodemográfico das pessoas intoxicadas.

\section{MÉTODOS}

Trata-se de uma pesquisa de cunho exploratório quantitativo, do ponto de vista do método, e transversal, no que se refere ao tempo de seguimento de estudo. Foram avaliadas as notificações compulsórias de intoxicação exógena do Piauí informada pelo Centro de Informação Toxicológica (CITOX) ao SINAN (Sistema de Informação de Agravos de Notificação) e DATASUS (Departamento de Informática do SUS) nos anos de 2013 a 2017.

Os dados coletados foram tabulados no programa Microsoft Excel versão 2016. As variáveis analisadas foram sexo, idade, zona de residência, agente tóxico, circunstância e classificação final, e confrontados com artigos científicos sobre o tema. Posteriormente, os dados foram avaliados através do programa estatístico BIOESTAT 5.0. Foi verificada a normalidade dos dados através do teste Shapiro Wilk e, posteriormente, foi aplicado o teste não paramétrico de Kruskal-Wallis. O nível de confiança adotado em todas as análises foi de $95 \%$.

\section{RESULTADOS E DISCUSSÃO}

A Tabela 1 ilustra o número de intoxicações exógenas por zona residencial, demonstrando que a maioria são oriundas da zona urbana. O Gráfico 1 expõe as intoxicações exógenas conforme a faixa etária, demonstrando que em todos anos analisados nesse estudo a faixa etária de maior prevalência foi a de 20-39 anos. Subsequentemente, no Gráfico 2 podemos evidenciar que no sexo feminino ocorreu maior predominância de intoxicações exógenas no período analisado nesta pesquisa. 
Tabela 1 - Intoxicações exógenas conforme a zona residencial no período de 2013 a 2017 no Piauí.

\begin{tabular}{|c|c|c|c|c|c|c|}
\hline Zona de residência & 2013 & 2014 & 2015 & 2016 & 2017 & Total \\
\hline Urbana & 653 & 840 & 889 & 1142 & 1310 & 4834 \\
\hline Rural & 186 & 219 & 219 & 298 & 261 & 1183 \\
\hline Periurbano & 1 & 0 & 1 & 2 & 1 & 5 \\
\hline Ign/brancos & 14 & 12 & 10 & 29 & 22 & 87 \\
\hline Total Anual & 854 & 1071 & 1119 & 1471 & 1594 & 6109 \\
\hline
\end{tabular}

Fonte: Silva ESF, et al., 2019.

Gráfico 1 - Intoxicações exógenas conforme faixa etária no período de 2013 a 2017 no estado do Piauí.

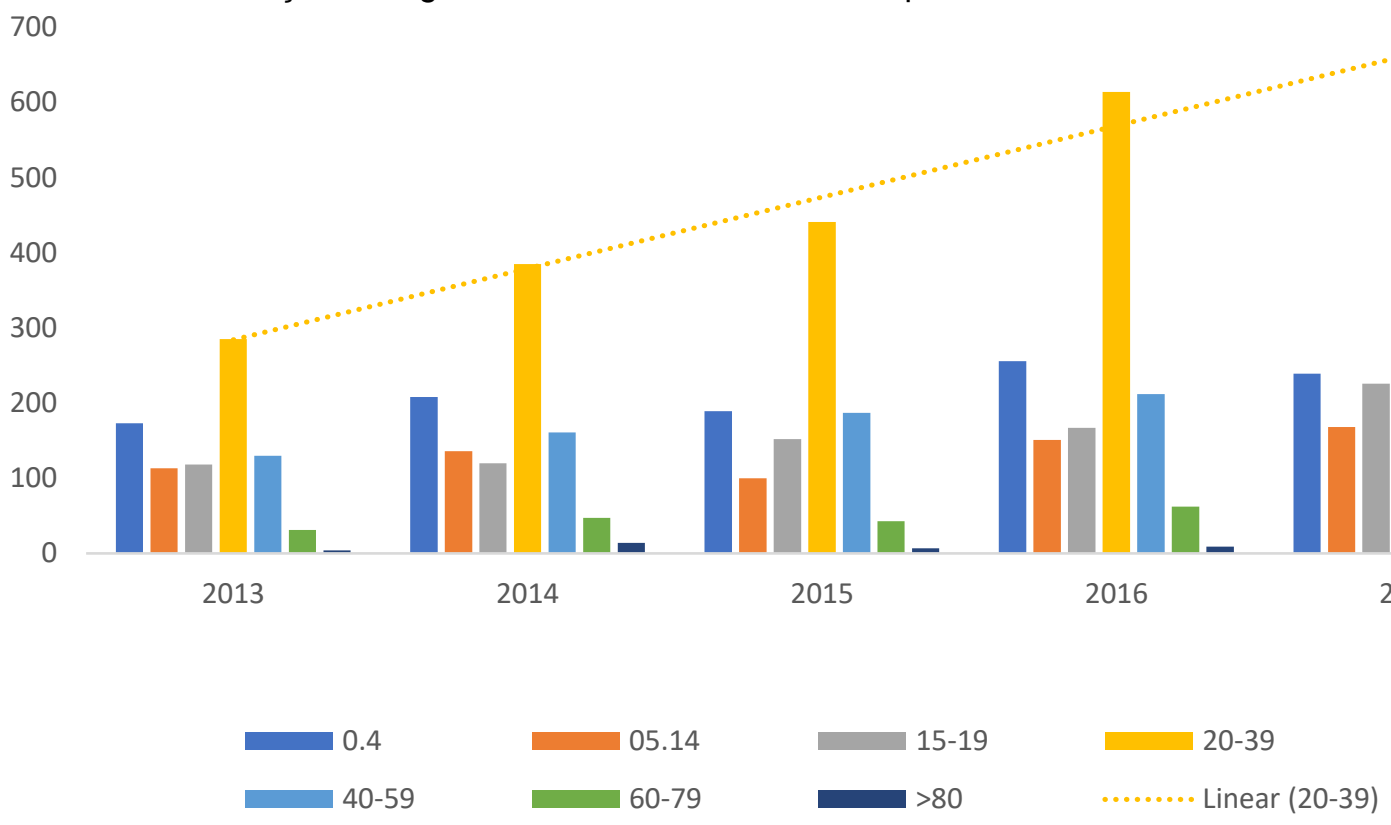

Fonte: Silva ESF, et al., 2019.

Gráfico 2 - Intoxicações exógenas conforme o sexo no período de 2013 a 2017 no estado do Piauí.

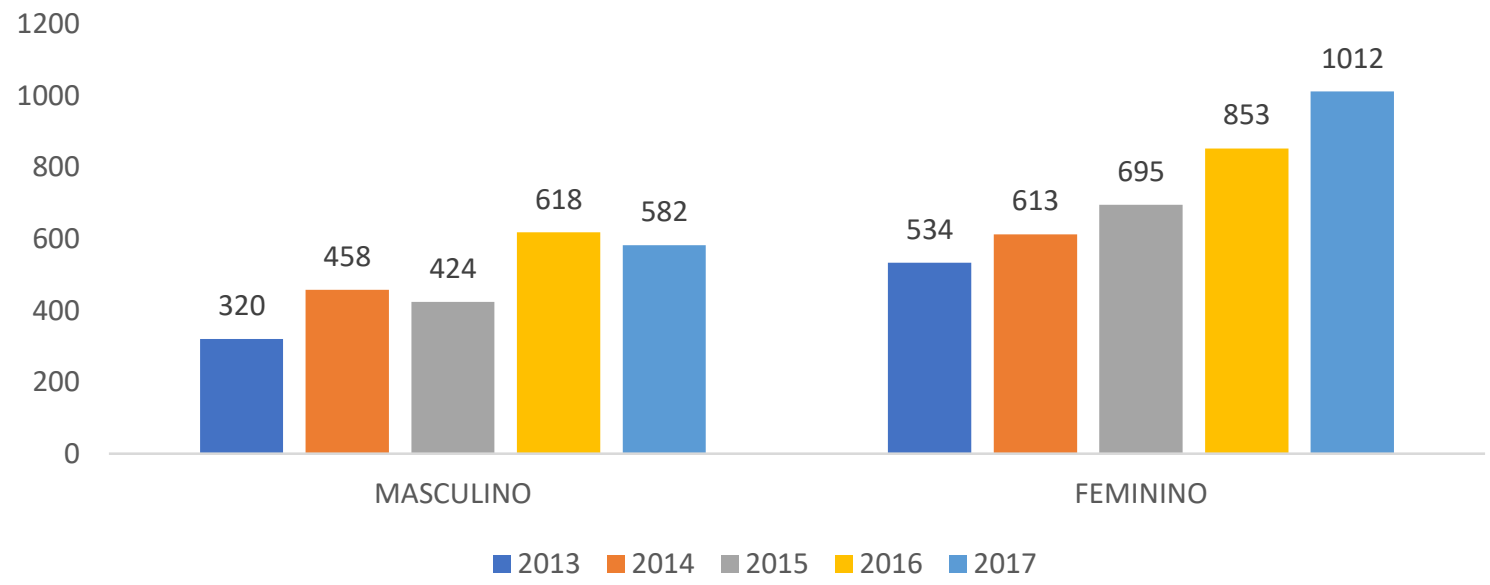

Fonte: Silva ESF, et al., 2019.

A Tabela 2 apresenta as intoxicações exógenas por agentes tóxicos mais frequentes no estado do Piauí no período de 2013 a 2017, apontando o medicamento como o agente tóxico mais frequente com 3252 notificações, alimentos e bebidas com 449, produto de uso domiciliar com 391, raticida com 262 e drogas de abuso com 189 também estão presentes. 
Tabela 2 -Intoxicações exógenas mais prevalentes segundo os Agentes tóxicos no período de 2013 a 2017 no estado do Piauí.

\begin{tabular}{lcccccc}
\hline \multicolumn{1}{c}{ Agente tóxico } & $\mathbf{2 0 1 3}$ & $\mathbf{2 0 1 4}$ & $\mathbf{2 0 1 5}$ & $\mathbf{2 0 1 6}$ & $\mathbf{2 0 1 7}$ & Total \\
\hline Medicamento & 435 & 526 & 610 & 781 & 900 & 3252 \\
Agrotóxico agrícola & 22 & 23 & 22 & 39 & 44 & 150 \\
Agrotóxico doméstico & 23 & 15 & 12 & 21 & 24 & 95 \\
Raticida & 43 & 49 & 46 & 65 & 59 & 262 \\
Produtos veterinários & 13 & 18 & 13 & 19 & 17 & 80 \\
Produtos químicos & 28 & 42 & 35 & 35 & 44 & 184 \\
Produtos uso domiciliar & 41 & 79 & 79 & 108 & 84 & 391 \\
Cosméticos & 11 & 21 & 26 & 28 & 25 & 111 \\
Drogas de abuso & 15 & 16 & 17 & 53 & 88 & 189 \\
Plantas tóxicas & 14 & 9 & 10 & 15 & 17 & 65 \\
Alimentos e bebidas & 49 & 74 & 77 & 146 & 103 & 449 \\
Outros & 15 & 19 & 19 & 52 & 96 & 201 \\
\hline \multicolumn{1}{c}{ Total anual } & 854 & 1071 & 1119 & 1471 & 1593 & 6109 \\
\hline
\end{tabular}

Legenda: ${ }^{*} \mathrm{p}=0,0054$.

Fonte: Silva ESF, et al., 2019.

A Tabela 3 Mostra as intoxicações exógenas por medicamentos originados do cruzamento com as circunstâncias mais evidenciadas no Estado do Piauí e a tentativa de suicídio como a principal circunstância, com uma frequência de 2312 dos casos ocorridos no último quinquênio, sucedido por acidental 1155 casos e uso terapêutico 435 casos, dentre outros. No Gráfico 3 é apresentada as intoxicações exógenas por medicamentos segundo a classificação final, no qual em todos os anos a maioria dos foram confirmados como intoxicação.

Tabela 3 - Distribuição de intoxicações exógenas por medicamentos segundo as circunstâncias no Estado do Piauí de 2013 a 2017.

\begin{tabular}{lcccccc}
\hline \multicolumn{1}{c}{ Circunstâncias } & $\mathbf{2 0 1 3}$ & $\mathbf{2 0 1 4}$ & $\mathbf{2 0 1 5}$ & $\mathbf{2 0 1 6}$ & $\mathbf{2 0 1 7}$ & Total \\
\hline Uso habitual & 45 & 92 & 62 & 169 & 61 & 429 \\
Acidental & 206 & 243 & 207 & 250 & 249 & 1155 \\
Ambiental & 6 & 11 & 6 & 5 & 5 & 33 \\
Uso terapêutico & 32 & 86 & 74 & 115 & 128 & 435 \\
Prescrisção médica & 1 & 2 & 1 & 2 & 2 & 8 \\
Erro de administração & 20 & 14 & 20 & 18 & 14 & 86 \\
Automedicação & 32 & 39 & 45 & 45 & 45 & 206 \\
Abuso & 20 & 17 & 40 & 58 & 86 & 221 \\
Tentativa de suicídio & 300 & 346 & 425 & 550 & 691 & 2312 \\
Violência/homicídio & 4 & 5 & 15 & 75 & 111 & 210 \\
Ingestão de alimentos & 42 & 45 & 61 & 56 & 71 & 275 \\
\hline \multicolumn{1}{c}{ Total anual } & 854 & 1071 & 1119 & 1471 & 1594 & 6109 \\
\hline
\end{tabular}

Legenda: ${ }^{*} \mathrm{p}=0,0086$.

Fonte: Silva ESF, et al., 2019. 
Gráfico 3 - Distribuição das intoxicações exógenas por medicamentos conforme a sua classificação final no Estado do Piauí, no período de 2013 a 2017.

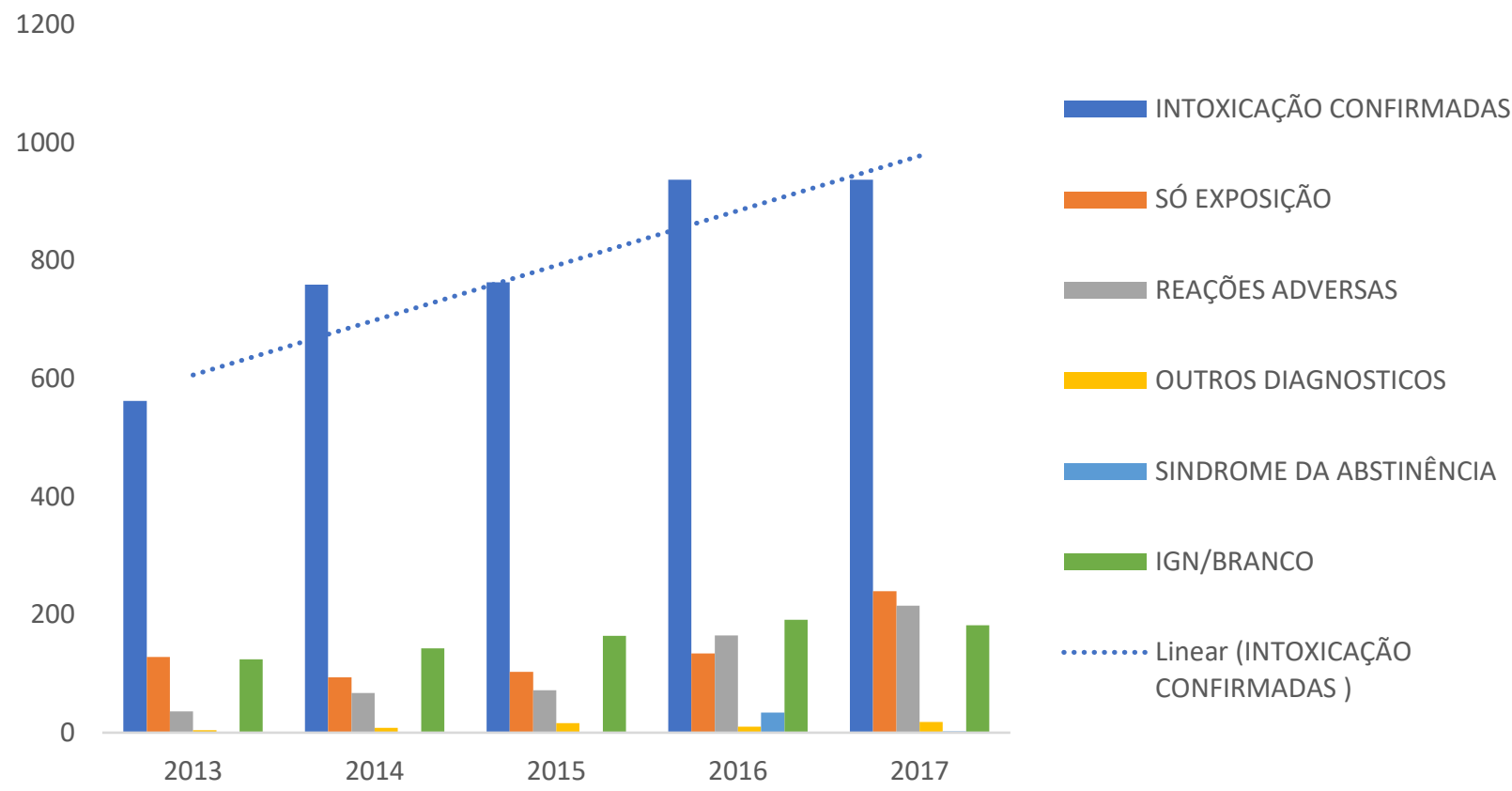

Fonte: Silva ESF, et al., 2019.

Diante dos resultados apresentados, foi evidenciado que as intoxicações têm maior incidência no perímetro urbano (Tabela 1), sendo o ano de 2016 o com maior número de ocorrências (1.139 casos). Monte BS, et al. (2016) sugere que isso se deve a maior facilidade de acesso aos medicamentos e às informações sobre eles, levando a automedicação ou uso indevido. A capital Teresina é a cidade com o maior número de casos, seguido de Floriano e Picos, vale ressaltar que estas cidades supracitadas, apresentam a maior economia do estado e estão entre os municípios com maior número de habitantes, o que está totalmente relacionado com o grande número de intoxicações que estas demostraram.

Analisando a variável faixa etária, pode-se observar que nos últimos 5 anos houve uma maior prevalência de intoxicações na população adultos jovens com idade entre 20-39 anos, com 2370 casos (Gráfico 1), seguido de intoxicações na faixa etária de 0 a4 anos, com 1065 casos, e a menor parte da população intoxicada possuía idade $>80$ anos com 47 notificações. Monte BS, et al. (2016) também encontrou resultados semelhantes em seu estudo, apresentando como justificativa o fato de os adultos jovens estarem no grupo de risco para acidentes com fármacos. Um estudo realizado por Escórcio MKB, et al. (2015) também encontrou a mesma faixa etária como sendo a mais prevalente nas intoxicações exógenas.

Ao se analisar o sexo das pessoas intoxicadas, observou-se que o feminino era maioria, com cerca de 3700 casos totais nos 5 anos analisados, contra aproximadamente 2400 do sexo masculino (Gráfico 2). A variável em debate está de acordo com uma pesquisa anterior de Oliveira GEG, et al. (2010), cujos achados foram de $50,1 \%$ dos casos em mulheres nos anos de 2007 a 2012. Na maioria dos casos, isso ocorre em tentativas de suicídio, como constatado pela Tabela 3 da presente pesquisa. De acordo com Kaplan $\mathrm{HI}$ et al. (1997), o sexo feminino é quatro vezes mais propenso a tentar o suicídio do que o sexo masculino. Os homens cometem suicídio com uma frequência três vezes maior do que as mulheres, os métodos utilizados na tentativa de suicídio diferem entre os sexos. Os homens tendem a usar meios mais violentos do que as mulheres, ao mesmo tempo em que as mulheres tendem a tomar doses excessivas de medicamentos ou de venenos (STACK S, 2000).

Através dos resultados da Tabela 2, visualiza-se que os medicamentos representam a maior causa de intoxicações exógenas no estado do Piauí, embora sejam utilizados na melhora e na atenuação dos efeitos das mais diversas doenças, eles também possuem seus efeitos nocivos quando utilizados de forma 
excessivos ou irregular, ou simplesmente devido a alergias ou intolerâncias desenvolvidas pelos pacientes (XAVIER LA, et al. 2017). Considera-se o quadro de mortalidade por intoxicação medicamentosa no Brasil como o reflexo do padrão de consumo dos medicamentos somado a fenômenos sociais, dentre os quais se pode citar: desigualdades, pobreza, desemprego e a tentativas de suicídio (MOTA DM, et al., 2012).

No estudo evidencia-se a presença de outros agentes tóxicos como causa de intoxicações, que possuem grande semelhança os medicamentos, pelo fato de dispor de constituintes farmacológicos, como raticidas, plantas tóxicas e drogas de abuso, porém apresentam mais efeitos negativos do que positivos, diferindo assim dos medicamentos (FIOCRUZ, 2010).

A pesquisa ressalta ainda que nos últimos 5 anos a classificação final dos casos teve $64,79 \%$ (3958) das notificações confirmadas por intoxicação. Observa-se também muitos casos de exposição e reações adversas, sendo respectivamente $11,44 \%$ e $9,1 \%$ dos casos. Contudo, os casos ignorados e brancos somam quase $13 \%$ do total das notificações compulsórias nos anos de 2013 a 2017 . Uma pesquisa trazendo informações relacionadas a exposição observou que a classificação final dos casos obteve $79,9 \%$ das notificações com intoxicação confirmada, $29,9 \%$ apenas exposição e $0,1 \%$ apresentaram reações adversas (TAKAHAMA CH, 2014), tais dados corroboram o encontrado nesta pesquisa, onde a grande maioria dos casos evidenciou intoxicação confirmada (Gráfico 3).

O número de casos de intoxicações vem aumentando no Brasil nos últimos cinco anos e no estado do Piauí os dados estatísticos acompanham o crescimento nacional, representando menos de $2 \%$ anuais em todo o país. Ocorrem em média 89.000 casos de intoxicações exógenas por ano em todo o Brasil, segundo o Sistema Nacional de Informações Tóxico-Farmacológicas (SINITOX). Os dados estatísticos encontrados fornecem subsídio para o conhecimento do perfil epidemiológico e elaboração de indicadores e planejamento de ações em saúde (MOTA DM, et al., 2012).

\section{CONCLUSÃO}

O estudo evidenciou que nos anos de 2013 a 2017 foram registrados cerca de 6000 casos de intoxicação exógena no Piauí, sendo os medicamentos os agentes tóxicos mais prevalentes e a tentativa de suicídio a principal circunstância entre jovens do sexo feminino. Esses dados demonstram a relevância desse estudo, visto que são considerados graves e preocupantes, apontando a importância de medidas de prevenção de intoxicações, uso racional de medicamentos e da promoção de práticas educativas para a conscientização da população.

\section{REFERÊNCIAS}

1. CHAVES LHS, et al. Intoxicação exógena por medicamentos: aspectos epidemiológicos dos casos notificados entre 2011 e 2015 no Maranhão. Reon Facema 2017; 3(2):477-482.

2. CITOX - Centro de Informação Toxicológica. Disponivel em: http://www.saude.pi.gov.br/divisa/citox. Acesso 24 mar. 2018.

3. ESCÓRIO MKBM, SOUSA AMC, RODRIGUES LM, et al. Análise dos casos notificados por intoxicação exógena no Piauí durante o período de 2010 a 2013. SANARE 2015; 14(s1).

4. FIOCRUZ- Fundação Oswaldo Cruz. Sistema nacional de informações tóxico-farmacológicas (SINITOX). Casos registrados de intoxicação humana por agente tóxico e centro, 2010. Disponível em: http://www.fiocruz.br/sinitox_novo/media/nd1.pdf. Acesso 12 set 2018.

5. GETTER CJ, NUNES JRDAS. Ocorrência de Intoxicações por Plantas Tóxicas no Brasil. Engenharia Ambiental $2011 ; 8(1)$.

6. HOCHMAN B, et al. Desenhos de pesquisa. Acta Cirúrgica Brasileira, v. 20. São Paulo, 2005.

7. KAPLAN HI, SADOCK BJ, GREBB JA. Compêndio de psiquiatria: ciências do comportamento e psiquiatria clínica. $7^{\mathfrak{a}}$ ed Porto Alegre: Artmed; 1997.

8. MAGALHÃES AFA. Avaliação das intoxicações exógenas do distrito federal no período de 2009 a 2013. [tese]BrasíliaDF: Universidade de Brasília; 2017.

9. MENDONÇA REB. O cuidado farmacêutico na prevenção da intoxicação medicamentosa. [monografia] Brasília-DF: 
Universidade Católica de Brasília;2012.

10. MONTE BS, et al. Estudo epidemiológico das intoxicações por medicamentos registrados pelo centro de informações toxicológicas do Piauí: 2017 a 2012. Revista interdisciplinar 2016; 9(3) 96-104.

11. MOREIRA CDAS, et al. Análise retrospectiva das intoxicações admitidas no hospital universitário da UFJF no período 2000-2004. Ciência e Saúde Coletiva 2010;15(3).

12. MOTA DM, et al. Perfil da mortalidade por intoxicação com medicamentos no Brasil, 1996-2005: retrato de uma década. Ciência \& saúde coletiva. 17(1):61-70

13. NOTIFICAÇÃO DE VIOLÊNCIA INTERPESSOAL/ AUTOPROVOCADA - Portaria GM/MS № 1271/2014 E SINAN: 2018. Ministério Da Saúde. [Acesso 29 mar. 2018] Disponível em: http://portalms.saude.gov.br/saude-de-az/acidentes-e-violencias/notificacao-de-violencia-interpessoal

14. OLIVEIRA GEG, et al. Análise das intoxicações por medicamentos em Picos-PI. Revista interdisciplinar de ciências médicas 2010.

15. RAMOS TO, COLLI VC, SANCHES ACS. Indicadores epidemiológicos das intoxicações exógenas em crianças menores de 5 anos na região de Araçatuba-SP. Revista Intertox de Toxicologia, Risco Ambiental e Sociedade2017; 30(3).

16. SILVA GCRF. O Método Científico Na Psicologia: Abordagem Qualitativa E Quantitativa2010. ok

17. Sistema Nacional De Informações Tóxico-Farmacológicas. [acesso 28 mar. 2018] Disponível em: https://sinitox.icict.fiocruz.br/sites/sinitox.icict.fiocruz.br/files//Brasil3_0.pdf

18. STACK S. Suicide: a 15-year review of the sociological literature. Part II: modernization and social integration perspectives. Suicide Life Threat Behav. 2000; 30(2):163-76. PMid:10888056.

19. TAKAHAMA CH, TURINI CA, GIROTTO E. Perfil das exposições a medicamentos por mulheres em idade reprodutiva atendidas por um Centro de Informações Toxicológicas. Ciênc. saúde coletiva 2014;19(4).

20. XAVIER LA, et al. Intoxicações exógenas por agentes tóxicos em crianças em município do norte de Minas Gerais. Revista Eletrônica Acervo Saúde 2017. 5s:s481-s485. 